\title{
ROTINAS ORGANIZACIONAIS NO AMBIENTE DA TEORIA EVOLUCIONÁRIA
}

\section{RESUMO}

A obra "Uma Teoria Evolucionária da Mudança Econômica" de Nelson e Winter (2005), além de importantes contribuições para os estudos organizacionais em geral, apresenta as bases de um debate abrangente acerca do conceito de Rotinas Organizacionais (RO). Nesta resenha, destaca-se o papel do conceito de RO como um dos fundamentos da teoria evolucionária, cujo pressuposto é que uma organização pode, a qualquer momento, incorporar maneira de fazer coisas e determinar o que deve ser feito, contrariando as noções ortodoxas de aptidões e de escolhas. Na perspectiva da Teoria Evolucionária, o conceito de RO apropria juntamente com a estabilidade própria a uma rotina, uma dimensão dinâmica necessária à sobrevivência no ambiente hostil do mercado. Após a apresentação de alguns dos fundamentos do conceito de RO associados à obra em foco, esta resenha insere ao debate a contribuição de outros estudos e autores sobre o tema. Finalmente, uma revisão da literatura recente sobre o tema, a partir da fonte Web of Science, evidencia ainda mais a oportunidade e a pertinência do conceito no atual ambiente empresarial.

Palavras chaves: Rotinas Organizacionais; Comportamentos Empresariais; Teoria Evolucionária; Estudos Organizacionais.

\section{ORGANIZATIONAL ROUTINES IN THE ENVIRONMENT OF THE EVOLUTIONARY THEORY}

\begin{abstract}
Nelson and Winter (2005) "An Evolutionary Theory of Economic Change", as well as important contributions to organizational studies in general, provide the basis for a comprehensive debate about the concept of Organizational Routines. In this review, we highlight the role of the RO concept as one of the foundations of evolutionary theory, whose assumption is that an organization can at any moment incorporate way of doing things and determine what should be done, contrary to the orthodox notions of aptitudes and choices. In the perspective of evolutionary theory, the RO concept appropriates, together with its own stability to a routine, a dynamic dimension necessary for survival in the hostile environment of the market. After presenting some of the fundamentals of the RO concept associated with the work in focus, this review associates the contribution of other studies and authors with the topic to the debate. Finally, a review of recent literature on the subject from the Web of Science source further highlights the timeliness and relevance of the concept in today's business environment.
\end{abstract}

Keywords: Organizational Routines; Business Behaviors; Evolutionary Theory, Organizational Studies. 


\section{ROTINAS ORGANIZACIONALES EN EL MEDIO AMBIENTE DE LA TEORÍA EVOLUCIONARIA}

\section{RESUMEN}

La obra "Una Teoría Evolucionaria del Cambio Económico" de Nelson e Invierno (2005), además de importantes contribuciones a los estudios organizacionales en general, presenta las bases de un debate exhaustivo sobre el concepto de Rutinas Organizacionales (RO). En esta reseña, destacamos el papel del concepto de RO como uno de los fundamentos de la teoría evolutiva, cuyo supuesto es que una organización puede en cualquier momento incorporar manera de hacer cosas y determinar qué debe hacerse, contrariando las nociones ortodoxas de aptitudes y de elecciones . En la perspectiva de la Teoría Evolucionaria, el concepto de RO apropia, junto con la estabilidad propia a una rutina, una dimensión dinámica necesaria para la supervivencia en el ambiente hostil del mercado. Después de la presentación de algunos de los fundamentos del concepto de RO asociados a la obra en foco, esta reseña asocia al debate la contribución de otros estudios y autores sobre el tema. Finalmente, una revisión de la literatura reciente sobre el tema a partir de la fuente Web of Science, evidencia aún más la oportunidad y pertinencia del concepto en el actual ambiente empresarial.

Palabras claves: Rutinas Organizacionales; Comportamiento Empresarial; Teoría Evolucionaria; Estudios Organizacionales.

Carlos Alberto Xavier do Nascimento ${ }^{1}$ Roberto Lima Ruas ${ }^{2}$ Dirceu da Silva ${ }^{3}$

\footnotetext{
${ }^{1}$ Doutorando pelo Programa de Pós-Graduação em Administração da Universidade Nove de Julho - PPGA/UNINOVE. Professor da Universidade Federal de Goiás - UFG. Brasil. E-mail: cnascimento3@hotmail.com

${ }^{2}$ Doutor em Economia pelo Université Picardie Jules Verne, França. Professor da Universidade Nove de Julho UNINOVE. Brasil. E-mail: robertoruas@uni9.pro.br

${ }^{3}$ Doutor em Educação pela Universidade de São Paulo - USP. Professor da Universidade Estadual de Campinas UNICAMP. Brasil. E-mail: dirceuds@gmail.com
} 


\section{INTRODUÇÃO}

A obra de Nelson e Winter $(2005)^{4}$, intitulada 'Uma teoria evolucionária da mudança econômica', tornou-se um clássico para os estudos organizacionais. Entretanto, as motivações iniciais para a construção da obra foram diferentes. Se por um lado, Nelson se preocupava com os processos de desenvolvimento a longo prazo, concentrando sua atenção na mudança tecnológica como força motora e na influência da política sobre o poder e direção dessa força, Winter entendia que a questão central residia no poder e nas limitações dos argumentos evolucionários que padronizavam a visão acerca do comportamento das firmas. (Nelson \& Winter, 2005).

A partir dessas premissas, pode-se compreender a construção das referências sobre rotinas organizacionais e sua contribuição para o avanço da teoria organizacional. Quando do desenvolvimento da obra, os autores introduzem a expressão "rotina", como um dos conceitos básicos da teoria evolucionária, admitindo que uma organização pode a qualquer momento incorporar maneiras de fazer coisas e determinar o que deve ser feito, contrariando as noções ortodoxas de aptidões e de escolhas.

Nelson e Winter (2005) alertam que entender o comportamento da firma, expresso na forma de rotinas, não é o mesmo que afirmar que suas principais atividades são imutáveis, ineficientes ou irracionais. Mas por outro lado, também consideram que a categoria de atividades desenvolvidas por uma empresa merece status conceitual diferente, pois reconhecem que flexibilizar um comportamento de rotina possui limitações, dado que, ao tentar modificá-las, a firma coloca em risco sua sobrevivência.

Essas observações são ainda mais oportunas atualmente num ambiente muito dinâmico, no qual as empresas precisam adaptar-se às mudanças econômicas, tecnológicas, políticas e sociais. Entretanto, carregam consigo o 'peso' e as 'virtudes' de sua trajetória empresarial (path dependence). Esse contraditório se efetiva nas rotinas organizacionais, naquilo que se pode denominar paradoxo da interação entre estabilidade e mudança e que já apresenta(m) correspondência no conceito de dynamic routines (Feldman, Pentland, D'Adderio, \& Lazaric, 2016)

Como se pode observar por esta breve introdução, a contribuição de Nelson e Winter (2005) na configuração do conceito de Rotinas Organizacionais (RO) continua muito atual e pertinente, apesar das mudanças revolucionárias no ambiente econômico e empresarial. Esta constatação

${ }^{4}$ Obra traduzida pela editora Unicamp (2005), a partir do original Nelson, R. R., \& Winter, S. G. (1982). An Evolutionary Theory of Economic Change. Cambridge, MA: Harvard University Press. evidencia-se no volume de publicações recentes levantadas na fonte Web of Science, conforme resultado apresentado no decorrer desta resenha.

Apesar das dificuldades para apropriar de maneira sucinta os principais elementos teóricos associados ao conceito de RO, considerando-se sua abrangência e complexidade (Becker, 2002), a trajetória desta resenha busca destacar aqueles elementos que parecem mais afinados à evolução do conceito no presente contexto. A partir da obra "Uma Teoria Evolucionária da Mudança Econômica", (Nelson \& Winter, 2005), foi contextualizado o conceito de RO. Após uma breve análise desses fundamentos, são consideradas algumas das contribuições de outros autores. Ao final, são apresentados os resultados de uma pesquisa acerca das publicações sobre o tema, no período 2009 -2016.

O estudo compreende mais três seções, além desta Introdução. Na seção 2, são apresentados e debatidos alguns dos fundamentos do conceito RO e suas características mais relevantes, a partir de Nelson e Winter (2005); na seção 3 são apresentadas algumas das implicações da obra em outros estudos posteriores e, por fim, na seção 4 , é a vez das considerações finais.

\section{AS ROTINAS ORGANIZACIONAIS SEGUNDO NELSON \& WINTER (2005) \\ 2.1 Fundamentos das RO no ambiente da Teoria Evolucionária}

Nesta seção, pretende-se destacar em quais aspectos as RO ajustam-se à proposta da Teoria Evolucionária, a partir da qual Nelson e Winter (2005) pretendem explicar o comportamento das empresas em ambiente de mudança econômica em oposição ao que denominam 'teoria econômica ortodoxa'.

Ainda, segundo Nelson e Winter (2005), a abordagem ortodoxa considera os seguintes tipos de comportamento das empresas:

$\checkmark$ Hipótese Maximizadora: leva os analistas a tentarem descobrir porque a firma está fazendo algo com base numa avaliação de seus objetivos e de seu conjunto de escolhas;

$\checkmark$ Hipótese do equilíbrio: o verdadeiro valor do preço e, portanto, o verdadeiro comportamento dos atores - é determinado pelas condições de equilíbrio da oferta e da demanda;

$\checkmark \quad$ Essas hipóteses são sustentadas por instrumentos matemáticos e giram em torno de regras de decisão;

Também para os autores, essa ortodoxia representa uma formalização e uma interpretação moderna da tradição mais ampla do pensamento 
econômico ocidental, cujas bases intelectuais podem ser traçadas a partir de "Smith; Marshall e Walras" (Nelson \& Winter, 2005). Os aurores, alertam tratarse de uma ortodoxia teórica, a qual preocupa-se diretamente com os métodos de análises econômicas e apenas, indiretamente, com quaisquer questões específicas às empresas.

Já a Teoria Evolucionária considera "as regras de decisão" como parentes conceituais muito próximos das "técnicas de produção", ao contrário da ortodoxia que as vê como dinâmicas diferentes. Dessa maneira, a "rotina" apresenta um papel importante na lógica da Teoria Evolucionária, o que inclui rotinas técnicas para a produção de coisas, procedimentos para contratações e demissões, encomendas de novos estoques, políticas relativas a investimentos e a pesquisa em $\mathrm{P} \& \mathrm{D}$ ou em publicidade etc. Na teoria evolucionária, as rotinas assumem a função que os genes apresentam na teoria evolucionária biológica. São características persistentes do organismo e determinam seu comportamento possível. Assim, na Teoria Evolucionária, os padrões comportamentais regulares e previsíveis da firma são considerados "rotinas", sendo essas moldadas num processo evolucionário específico a cada empresa.

$\mathrm{Na}$ obra em foco, Nelson e Winter (2005) enfatizam que a utilização do termo 'Teoria Evolucionária' para descrever uma alternativa à ortodoxia, requer ainda um debate consolidador, pois sinalizam o empréstimo que faz das ideias centrais da Biologia Darwiniana, especialmente a aplicação na economia do princípio da "seleção natural".

De fato, a associação do mercado como ambiente pleno de desafios e armadilhas no qual a sobrevivência das espécies - no caso os diferentes tipos de empresas - se torna um grande desafio de sobrevivência e expansão. Nesse contexto, a princípio, parece uma analogia muito inspiradora.

A partir dessa construção, desponta a visão de "genética organizacional", no qual processos (ou rotinas) e suas características organizacionais, incluindo habilidades de gerar produtos e auferir lucros, são transmitidos ao longo do tempo. Nessa ótica, relatam os autores, que as conotações mais amplas do termo "evolucionário" incluem preocupação processual de mudanças a longo prazo e progressivos. Rotinas podem então ser entendidas como regularidades observadas no presente, associadas a "resultados produzidos por processos dinâmicos compreensíveis" (Nelson \& Winter, 2005, p.26), sustentados em realidades construídas ao longo de trajetórias passadas.

Também Nelson e Winter (2005) destacam que a abordagem evolucionária compreende também "desenvolvimento gradual", expressão associada à interação entre os termos "evolucionárias" e "revolucionárias", pois não negam que por vezes as mudanças possam ser muito rápidas. Por outro lado, os autores contestam qualquer intenção de perseguir a analogia biológica, ou mesmo, seguir em direção a uma teoria abstrata e de nível superior que seja capaz de incorporar outras teorias, inclusive a Economia. Destacam que identificaram na Biologia ideias capazes de serem ajustadas analogicamente à problemas econômicos.

Enfim, é nesse composto teórico que os autores definem o termo "rotinas" como padrões comportamentais regulares e previsíveis das firmas. Segundo Nelson e Winter (2005), alguns exemplos de rotinas são:

- Rotinas técnicas especificadas para a produção de bens;

- Procedimentos para contratações e demissões;

- Encomendas de novos estoques;

- Rotinas para aumentar a produção de itens de alta demanda;

- Políticas relativas ao investimento, à pesquisa e desenvolvimento (P\&D) ou à publicidade;

- Estratégias empresariais relativas à diversificação da produção e ao investimento no exterior.

Assim, conforme a teoria evolucionária econômica postulada desses autores, essas rotinas assumem a função que os genes representam na Teoria Evolucionária biológica:

- São características persistentes do organismo e determinam seu comportamento possível, embora o comportamento real também seja determinado pelo meio ambiente;

- Elas são hereditárias no sentido de que os organismos de amanhã são gerados pelos de hoje, como, por exemplo, a construção de uma nova planta fabril preserva algumas características fundamentais que a empresa apresenta em outras plantas já existentes, mesmo considerando as melhorias implementadas;

- São selecionáveis no sentido de que organismos com certas rotinas podem sair-se melhor do que outros e, se assim for, sua importância relativa na população (no ramo de atividades) vai aumentando ao longo do tempo.

Os autores, porém, alertam para a existência de grande conjunto de comportamentos empresarias que não podem ser constituídos como 'rotinas', a exemplo de decisões empresarias importantes, seja do ponto de vista da firma individual, seja do ponto de vista da sociedade. (Nelson \& Winter, 2005, p. 33).

De fato, nem todo o comportamento empresarial pode seguir um padrão regular e previsível e, por isso, não pode ser acomodado na 
Rotinas Organizacionais no Ambiente da Teoria Evolucionária

"teoria evolucionária", já que se reconhece a existência de elementos estocásticos que dependem ou resultam de uma variável aleatória, seja na determinação das decisões, seja nos resultados das decisões. Trata-se de eventos difíceis de prever, e alertam os autores que, se assim não fosse, o observador veria-se tentado interpretar o tumulto e a sensação de crise como uma espécie de ritual organizacional ou uma espécie de rotina.

Nesse sentido, Nelson \& Winter (2005, pp. 36-37) apresentam três classes de rotinas as quais permitem analisar o processo evolucionário que as moldou:

a. Relaciona-se ao que a firma realiza a qualquer momento, considerando seu estoque anterior de plantas, equipamentos e outros fatores de produção que não podem ser rapidamente aumentados no curto-prazo, o que os autores denominam características operacionais;

b. Relaciona-se ao aumento ou diminuição do estoque de capital da firma, considerando-se os períodos (curto prazo), de maneira que o padrão a prevalecer pode ser dependente do porte do projeto de investimento, na atividade existente da firma. Nessa perspectiva, segundo os autores, a exemplo do que ocorre na teoria biológica, a sensibilidade da taxa de crescimento de uma firma é reflexo de seus "genes";

c. Relaciona-se à visão de que as empresas podem ser representadas por rotinas que expressam suas características operacionais ao longo do tempo, incluindo as mudanças observadas. Assim, empresas modelares na teoria evolucionária podem ser compreendidas como aquelas que apresentam departamentos capazes de analisar mercados, oficinas de pesquisa e laboratórios de pesquisa e desenvolvimento. E no caso de não apresentarem tais departamentos, é necessário que essas, ao menos periodicamente, engajem alguns profissionais para refletirem o que a empresa está fazendo e por quê, possibilitando uma revisão ou, até mesmo, uma mudança radical.

A preocupação central da teoria evolucionaria diz respeito aos processos dinâmicos que determinam conjuntamente os padrões de comportamentos da firma, bem como os resultados de mercado ao longo do tempo. Nesse aspecto, os autores destacam a transição de um período para outro, no qual valorizam as ações da empresa realizadas durante o caminho percorrido, sem esquecer que a situação do ramo de atividade em cada período também carregará sementes de sua situação no período seguinte. É nesse ambiente que as RO aparecem como elementos fundamentais. (Nelson \& Winter, 2005).

\subsection{Rotinas e Habilidades: a concretização das RO}

Embora os autores entendam que o fator habilidades não seja o único elemento que mobiliza o comportamento individual, consideram a analogia entre habilidades individuais e rotinas organizacionais elemento relevante para que se entenda a dinâmica das RO (Nelson \& Winter, 2005, p. 116):

A rotina é relativamente mais decisiva
como característica do comportamento da
organização do que a habilidade como
característica do comportamento do
indivíduo. Entretanto, em ambos os níveis,
um exame rigoroso da natureza das relações
entre comportamento, rotinas e habilidades
traz a luz os limites das noções de
otimização como uma abordagem para a
compreensão da base do funcionamento
eficiente de um indivíduo ou de uma
organização em dado contexto

Nelson e Winter (2005) definem habilidades como capacidades de ter uma sequência regular de comportamentos coordenados, o que, em geral, faz dele um comportamento eficiente em relação a seus objetivos. Destacam que as habilidades apresentam algumas características comuns às RO: primeiro, as habilidades são programáticas; segundo, um desempenho habilidoso constitui em grande parte um tipo de conhecimento tácito e; terceiro, exercitar determinada habilidade envolve fazer numerosas escolhas, embora muitas vezes não se tem a consciência de que se está fazendo escolhas. No entanto, esses três elementos do comportamento habilidoso são intimamente relacionados, segundo esses autores.

Ainda na busca de traçar uma relação entre habilidades e rotinas, discutem habilidades tácitas e habilidade de escolha e sobre essa última $\mathrm{e}$ argumentam que "todo comportamento sequencial coordenado envolvido no exercício de uma habilidade é um comportamento escolhido" e que em geral, o processo de escolha assume papel fundamental na seleção de grandes unidades de comportamento. O mesmo não ocorre na seleção de pequenas unidades de comportamento.

Ao tratar da habilidade do homem de negócios, Nelson e Winter (2005, p. 143), destaca que:

Um homem de negócios experiente que age à procura de ganho pecuniário é um 
indivíduo que está exercitando uma habilidade complexa. Como ocorre com qualquer habilidade desse tipo, a busca de ganho é baseada em conhecimento tácito sobre as condições relevantes e envolve uma consciência bastante subsidiária de muitos detalhes dos procedimentos que estão sendo seguidos.

Quanto às aptidões organizacionais, Nelson e Winter (2005) baseiam-se em teóricos e economistas como March \& Simon, Allison, Gouldner, Perrow, Doeringr \& Piore, Williamson, Schumpeter e outros, e por aí distinguem o tratamento dos autores da teoria evolucionária ao paralelismo traçado entre habilidades individuais e as rotinas organizacionais. Destacam os autores a grande variedade de organizações sendo improvável que um dado conjunto de conceitos e proposições possa ser aplicado de maneira uniforme e com utilidade a todos esses tipos. Distinguem os autores os seguintes tipos de organização:

a) Organizações comprometidas com o fornecimento de bens e serviços para algum cliente externo e que têm ao menos um vago critério do sobre o que é ter um desempenho bom ou mal; b) Por ser a "Rotina" conceito-chave do estudo, este se aplicaria com bastante naturalidade a organizações comprometidas com o fornecimento de bens e serviços que se mantenham aparentemente "as mesmas" ao longo de extensos períodos e,

c) A discussão reflete principalmente organizações tidas como "grandes e complexas" e a questão restritiva é simplesmente a de manter o foco nos fenômenos que são tipicamente organizacionais (p. 150).

Por fim, ainda cabe destacar os diferentes papéis do conceito de Rotina, apresentados no Quadro 1, o qual sintetiza as contribuições que a noção de rotinas pode trazer à reflexão acerca da gestão estratégica das organizações, o que encerra o recorte literário explorado no presente trabalho segundo as contribuições de Nelson e Winter (2005). Desta forma, fica registrado o debate pretendido pelos autores acerca das diferenças entre as teorias econômicas evolucionária e ortodoxa, explorando especialmente suas respectivas contribuições ao desenvolvimento das organizações.

\begin{tabular}{|c|c|}
\hline $\begin{array}{l}\text { PAPÉIS DAS } \\
\text { ROTINAS }\end{array}$ & CARACTERÍSTICAS \\
\hline $\begin{array}{l}\text { Rotina como } \\
\text { memória da } \\
\text { Organização }\end{array}$ & $\begin{array}{l}\text { Onde está o conhecimento? Onde está a memória de uma organização? Segundo os } \\
\text { autores, a "rotinização das atividades de uma organização constitui a forma mais } \\
\text { importante de estocagem do conhecimento específico a ela". }\end{array}$ \\
\hline Rotina como trégua & $\begin{array}{l}\text { Reconhecimento da divergência de interesse entre membros da organização e oferecem a } \\
\text { base de uma racionalidade pela qual as organizações, no entanto, podem ser modeladas } \\
\text { sem dar atenção explícita ao fato de haver o envolvimento de muitos participantes. das } \\
\text { diferenças entre seus membros. }\end{array}$ \\
\hline $\begin{array}{l}\text { Rotina como meta: } \\
\text { controle cópia e } \\
\text { Imitação } \\
\end{array}$ & $\begin{array}{l}\text { Operação de rotinas como objetivo dos esforços direcionados para o controle da } \\
\text { organização, para a reprodução das rotinas existentes e para imitação de rotinas } \\
\text { empregadas em outras organizações. }\end{array}$ \\
\hline $\begin{array}{l}\text { Rotinas e } \\
\text { habilidades: } \\
\text { comparações }\end{array}$ & Explicações do paralelismo entre organizações e habilidades dos indivíduos \\
\hline $\begin{array}{l}\text { Rotinas ótimas e } \\
\text { Rotinas de } \\
\text { Otimização }\end{array}$ & $\begin{array}{l}\text { Relação entre o conceito de operação rotineira e a reinvindicação da ortodoxia de que as } \\
\text { firmas otimizam. Também ao fato de que ao menos algumas das firmas empregam } \\
\text { métodos de otimização explícitos para tomar algum tipo de decisão em certas situações. }\end{array}$ \\
\hline $\begin{array}{l}\text { Rotinas, heurísticas e } \\
\text { inovação }\end{array}$ & $\begin{array}{l}\text { Explora a conexão entre comportamento rotinizado e o comportamento inovador, } \\
\text { enfatizando haver menos oposição entre essas duas ideias que comumente se supõe. }\end{array}$ \\
\hline
\end{tabular}

Quadro 1 - Papéis de Rotinas e Características

Fonte: adaptado de Nelson e Winter (2005, pp. 152 - 153) 
Rotinas Organizacionais no Ambiente da Teoria Evolucionária

\section{IMPLICAÇÕES DA OBRA DE NELSON E WINTER E OUTRAS ABORDAGENS SOBRE ROTINAS ORGANIZACIONAIS}

O estudo de Nelson e Winter (2005), denominado teoria evolucionária, constitui um marco teórico para se analisar o desenvolvimento observado no ambiente das firmas, a partir de uma perspectiva alternativa ao modelo baseado na economia ortodoxa. Nessa trajetória, esta resenha destaca a noção de rotinas organizacionais, a qual expressa padrões de comportamento nos processos internos às firmas. Esses padrões de comportamento são dependentes de sua trajetória (path-dependence); pois, segundo os autores, as rotinas são moldadas pela história e caminhos percorridos pelas empresas.

Essa noção de dependência à trajetória vai marcar a aplicação do conceito em investigações empíricas, valorizando as análises do tipo longitudinal, a fim de tentar compreender os processos de construção da rotina organizacionais. Nesse sentido, Levitt e March (1988) entendem que o histórico e as razões que levaram as empresas por um certo caminho não podem ser reconstruídos por outras empresas, tampouco os problemas que geraram a rotina original.

As rotinas também apresentam um papel importante na economia de recursos cognitivos e é possivelmente essa a razão de iniciativas visando à construção de rotinas nas organizações. Anteriormente a Nelson e Winter (2005), outros estudos apontavam que os recursos eram escassos e as organizações não teriam condições de atender a todos os seus objetivos simultaneamente. (Simon, 1947; Simon, 1955; March \& Olsen 1976). Outros estudos mostravam indicativos de que as rotinas reduzem custos de governança (Penrose 1952; Postrel \& Rumelt, 1992; Hodgson, 1997). As rotinas também podem reduzir incertezas, pois ao estabelecer um padrão de atuação, liberam recursos mentais para que os atores possam lidar com a complexidade e indecisões.

Uma das obras que recupera vários aspectos de Nelson e Winter (2005) é a de Becker (2002), na qual o autor tenta explicar os diversos sentidos que podem ser expressos no conceito de rotinas a partir da questão: no que consistem esses padrões de comportamento? Nesse caminho, aponta 4 elementos capazes de auxiliar na resposta: a) Ação, b) Atividade, c) Comportamento e d) Interação. (Becker, 2002).

Além de vasculhar esses elementos, Becker (2002) busca encontrar a dinâmica que é capaz de mediá-los: o termo "interação" conota uma dimensão coletiva; já a diferença entre "ação" e "interação" enfatiza a distinção entre o individual e o coletivo, o que resulta que esses padrões circulam por diferentes níveis: o individual e o coletivo, e o observável e o não-observável. Aliás, parece haver consenso de que as rotinas são fenômenos coletivos (Becker, 2002, p. 9). Assim sendo, o autor recorre a Weick (1990) ao revelar que a rotina pode ser interrompida quando os participantes agem de uma forma que é mais individual que coletiva.

Um outro aspecto importante em Nelson e Winter (2005) é a repetitividade/persistência. Becker (2002) entende que sendo a repetitividade elemento característico das rotinas, seria inconcebível uma rotina que não haja repetição, pois a condição de poucas mudanças é o que tornam as rotinas estáveis. Por outro lado, não há como não considerar os limites da repetitividade e persistência num ambiente instável e dinâmico pós-década de 80. Por isso, segundo Becker (2002), caracterizar a interação entre repetitividade e mudança no ambiente das rotinas depende do ambiente no qual se inserem as rotinas.

Outros estudos também apontam a dificuldade para precisar a dinâmica entre estabilidade e mudança nas RO, bem como para caracterizar os elementos que comporiam esse processo. (Cohen \& Bacdayan 1994; Cohen, Burkhart, Dosi, Egigi, Marengo, Warglien, \& Winter, 1996; Reynaud 1998; Jones \& Craven 2001).

Mas provavelmente, a contribuição mais relevante neste debate sobre estabilidade e mudanças das rotinas aparece em Feldman (2000) e Feldman e Pentland (2003), estudos nos quais os autores destacam, com base em investigação empírica, que o caráter estável e institucional das RO é alterado por uma dinâmica interna resultante da atuação dos participantes quando reagem às interações nas rotinas. Com base nessas investigações, os autores propõem que, ao lado do caráter institucional e ostensivo das rotinas, é preciso considerar seu caráter performativo resultante da atuação dos participantes da rotina.

Um outro debate relevante é o que trata da relação entre rotinas e conhecimento: "a rotinização da atividade em uma organização constitui a forma mais importante de armazenamento de conhecimento operacional específico da organização" (Nelson e Winter, 2005, p.99), e ainda, segundo eles, além de constituírem repositório de conhecimento na empresa, podem armazenar as soluções bemsucedidas para problemas específicos.

Outros estudos corroboram essa posição, reafirmando que a rotina seria o mais importante meio de incorporar conhecimento tácito. (Winter, 1987b; Winter, 1994; Teece, Rumelt, Dosi, \& Winter, 1994). Aliás, a questão da incorporação de conhecimento por meio das rotinas é tema de debate atual, constituindo parte da noção de capacidade absortiva, processo do qual uma das etapas, denominada assimilação ou internalização do conhecimento tem exatamente como papel principal, a apropriação de novos conhecimentos às rotinas da 
organização, a fim de viabilizar inovações (Zahara \& George, 2002).

Rotinas como "gatilhos" é outro campo de discussão presente na obra de Nelson e Winter (2005), ou seja, rotinas são acionáveis e, por isso, são capazes de desencadear outras rotinas. Mais adiante, Nelson (1994) reforça que rotinas tendem a se construírem quando certas maneiras de fazer as coisas de forma consistente trazem resultados pelo menos satisfatórios.

Duas outras questões também importantes e que se desdobram em outros estudos são o papel relevante dos gestores profissionais na geração, seleção e aplicação de rotinas organizacionais superiores (Knott \& McKelvey, 1999) e a importância das rotinas organizacionais na relação entre franqueados e franqueadores (Zollo \& Winter, 2002).

\subsection{Rotinas Organizacionais: contribuições no período 2009 - 2016}

A fim de precisar alguns dos avanços no debate acerca do conceito "rotinas organizacionais" este tópico investiga na base de dados do Web of Science, considerando: a) palavra-chave (Organizational Routines), b) Categoria (Management e Business), c) Tipos de documentos (Artigos), d) Área (Management e Business Economics), e) Título (Organizational Science, Organizational Studies e Jornal of Management Studies), f) ano de publicação (2009 a 2016) e por fim, linguagem (english and portuguese). A pesquisa junto à base de dados resultou em 78 (setenta e oito) artigos encontrados, cujas referências são destacadas no Quadro 02. 


\begin{tabular}{|c|c|c|}
\hline ANO & AUTORES & CONTRIBUIÇÃO \\
\hline \multirow{4}{*}{2009} & Salvato, Carlo & $\begin{array}{l}\text { Microatividades - Renovação organizacional e vantagem competitiva pode, portanto, exigir mudança parcial de foco de } \\
\text { capacidades como entidades agregadas. }\end{array}$ \\
\hline & Laamanen, Tomi; Wallin, Johan & $\begin{array}{l}\text { Dinâmicas cognitiva no desenvolvimento da cognição gerencial. Desenvolvimento de caminho. Capacidades dinâmicas. } \\
\text { Microfundamentos cognitivos de desenvolvimento de capacidades. }\end{array}$ \\
\hline & $\begin{array}{l}\text { Christianson, Marley K.; Farkas, Maria } \\
\text { T.; Sutcliffe, Kathleen M.; et al. }\end{array}$ & $\begin{array}{l}\text { Aprender por meio de eventos raros: interrupções significativas. Exploração da ligação entre a aprendizagem e eventos raros. } \\
\text { O problema não é tanto o que as organizaçôes aprendem "de" raros eventos, mas o que aprendem "por meio de" eventos raros. }\end{array}$ \\
\hline & $\begin{array}{l}\text { McKendrick, David G.; Wade, James } \\
\text { B.; Jaffee, J. }\end{array}$ & Cisão e desempenho tecnológico das empresas mãe. Efeitos decorrentes de tecnologia, spin-offs e rotinas. \\
\hline \multirow{8}{*}{2010} & Mathews, John A & Recursos, atividades, rotinas e desiquilíbrio. \\
\hline & Sullivan, Bilian Ni & Problemas. Alocação de Atenção no processo de regulamentação organizacional. \\
\hline & Hibbert, Paul; Huxham, Chris & O Passado em Jogo: Tradição nos organismos de colaboração. Colaboração interorganizacional e desafios complexos. \\
\hline & Csaszar, Felipe A.; Siggelkow, Nicolaj & $\begin{array}{l}\text { Quanto a copiar - Amplitude de Imitação. As práticas de imitação de empresas e os impactos nas empresas imitadas e as que } \\
\text { imitam. }\end{array}$ \\
\hline & Kane, Aimee A & $\begin{array}{l}\text { Potencial de transferência de conhecimento. Demonstrabilidade conhecimentos e impacto de identidade social superior. } \\
\text { Probabilidade de que as organizações capitalizam seus recursos de conhecimento transmitindo conhecimento na forma de uma } \\
\text { rotina de produção. }\end{array}$ \\
\hline & $\begin{array}{l}\text { Pentland, Brian T.; Haerem, Thorvald; } \\
\text { Hillison, Derek }\end{array}$ & Rotinas organizacionais como padrões recorrentes de Ação. \\
\hline & Zbaracki, Mark J.; Bergen, Mark & A trégua em Colapso! Microfundamentos da rotina em um estudo de processos de ajuste de preço. \\
\hline & $\begin{array}{l}\text { Eisenhardt, Kathleen M.; Furr, Nathan } \\
\text { R.; Bingham, Christopher B. }\end{array}$ & $\begin{array}{l}\text { Microfundamentos de desempenho. Equilíbrio da eficiência. Ambientes dinâmicos. Capacidades dinâmicas para o } \\
\text { desempenho e o papel dos líderes em gerenciar a tensão. }\end{array}$ \\
\hline \multirow{7}{*}{2011} & $\begin{array}{l}\text { Lewin, Arie Y.; Massini, Silvia; } \\
\text { Peeters, Carine }\end{array}$ & $\begin{array}{l}\text { Microfundamentos de rotinas. Capacidade interna e externa de absorção. A capacidade de absorção: uma nova perspectiva } \\
\text { sobre a aprendizagem e a inovação de administração. }\end{array}$ \\
\hline & Schreyoegg, G; Sydow, Joerg & Dependência da trajetória organizacional. Teoria dos processos e a estrutura organizacional. \\
\hline & Leonardi, Paul M. & $\begin{array}{l}\text { Inovação Cega. Tecnologia. Artefatos tecnológicos. Cultura. Consequências da "cegueira da inovação" no desenvolvimento de } \\
\text { conceitos de tecnologia através das fronteiras organizacionais e ocupacionais. }\end{array}$ \\
\hline & Nicolini, Davide & Introdução do conceito de "local" para ajudar a avançar a compreensão da relação entre a prática e saber. \\
\hline & Cabantous, Laure; Gond, Jean-Pascal & $\begin{array}{l}\text { Racionalidade na tomada de decisão. Teoria da escolha racional. Racionalidade na vida organizacional. Proposta de uma nova } \\
\text { perspectiva sobre a racionalidade que permite a teorização da produção de decisões racionais pelas organizações. }\end{array}$ \\
\hline & Brown, Andrew D.; Lewis, Michael A & Identidade. Disciplina e Rotinas. Identidades e rotinas organizacionais. \\
\hline & $\begin{array}{l}\text { Feldman, Martha S.; Orlikowski, } \\
\text { Wanda J. }\end{array}$ & anizacionais. Abordagens (empíricos, teóricos e filosóficos) e a relação com a prática. \\
\hline
\end{tabular}




\begin{tabular}{|c|c|c|}
\hline & Sminia, Harry & Análise da continuidade institucional. A análise utiliza uma abordagem prática baseada em estruturação de institucionalização. \\
\hline & $\begin{array}{l}\text { Lavie, Dovev; Kang, Jingoo; } \\
\text { Rosenkopf, Lori }\end{array}$ & $\begin{array}{l}\text { Equilíbrio dentro e entre domínios: as implicações de desempenho de exploração e aproveitamento em alianças. Desafios em } \\
\text { conciliar exploração e aproveitamento. Rotinas organizacionais conflitantes. }\end{array}$ \\
\hline & $\begin{array}{l}\text { Pentland, Brian T.; Hrem, Thorvald; } \\
\text { Hillison, Derek }\end{array}$ & $\begin{array}{l}\text { Padrão de ação. Prevê que as rotinas devem gerar padrões de ação que são poucos em número e estável ao longo do tempo, e } \\
\text { que os padrões atípicos de ação são impulsionados principalmente por insumos excepcionais. A teoria concorrente prevê o } \\
\text { oposto. Mudanças nem sempre refletem melhor desempenho ou de aprendizagem. Automação. }\end{array}$ \\
\hline \multirow{15}{*}{2012} & $\begin{array}{l}\text { Labatut, Julie; Aggeri, Franck; Girard, } \\
\text { Nathalie }\end{array}$ & $\begin{array}{l}\text { Implementação de tecnologia e seus efeitos disciplinares na interagem alteração de rotinas organizacionais. Capacidades dos } \\
\text { atores, produzir novos padrões de ação. Dualidade de rotinas organizacionais (ostensiva e aspectos performativos). }\end{array}$ \\
\hline & Turner, Scott F.; Rindova, Violina & $\begin{array}{l}\text { Consistência de funcionamento. Mudança em curso. Pressões de equilíbrio de rotinas. Aspectos ostensivos da rotina } \\
\text { operacional. Flexibilidade na coordenação interna, Artefatos e conexões. Perspectiva dinâmica em rotinas. }\end{array}$ \\
\hline & $\begin{array}{l}\text { Jarzabkowski, Paula A.; Le, }, \\
\text { Feldman, Martha S. }\end{array}$ & $\begin{array}{l}\text { Atividades Dinâmicas e coordenação. Coordenar as AD continuamente criados e modificados, a fim de promulgar } \\
\text { relacionamentos e atividades organizacionais. Organização e cadeia de valor. }\end{array}$ \\
\hline & Rennstam, Jens & $\begin{array}{l}\text { Problema do controle no trabalho de conhecimento intensivo. Processos criativos de conhecimento e habilitação na } \\
\text { organização. Quando o trabalho se torna complexo, os gerentes tentam controlar as normas e das identificaç̃̃es dos } \\
\text { funcionários, ao invés de seus comportamentos. }\end{array}$ \\
\hline & Currie, Graeme; White, Leroy & Intermediação do conhecimento situado dentro de um contexto organizacional, caracterizada pela hierarquia profissional. \\
\hline & $\begin{array}{l}\text { Anand, Gopesh; Gray, John; Siemsen, } \\
\text { Enno }\end{array}$ & Rotinas operacionais. Processos de entropia. Deterioração na adesão a rotinas operacionais ao longo do tempo. \\
\hline & Raman, Roopa; Bharadwaj, Anandhi & $\begin{array}{l}\text { Efeitos do Processo de transferência de prática e avanços. Desvios performativos. Papeis diferenciais de poder. Incerteza e } \\
\text { tecnologia da informação. }\end{array}$ \\
\hline & Obstfeld, David & Quadro de ação. Organizações, rotinas e "projetos criativos. Diferentes formas de mudança organizacional e inovação. \\
\hline & $\begin{array}{l}\text { Bapuji, Hari; Hora, Manpreet; Saeed, } \\
\text { Akbar M. }\end{array}$ & $\begin{array}{l}\text { Emergência das rotinas e desempenho organizacional. Intermediários transmitem as intenções de um ator para outro e, } \\
\text { portanto, potencialmente alinham as ações e respostas dos atores }\end{array}$ \\
\hline & Cacciatori, Eugenia & $\begin{array}{l}\text { Conflitos na resolução de problemas. Processo de desenvolvimento de novas rotinas. Anomalias no desenvolvimento de novas } \\
\text { rotinas. Artefatos individuais. }\end{array}$ \\
\hline & $\begin{array}{l}\text { Miller, Kent D.; Pentland, Brian T.; } \\
\text { Choi, Seungho }\end{array}$ & $\begin{array}{l}\text { Interação dinâmica entre ação e memória em rotinas organizacionais. Três formas distintas de memória de procedimento, } \\
\text { declarativa e transacional e examinam seus papéis na formação, eficiência e capacidade de adaptação das rotinas. }\end{array}$ \\
\hline & $\begin{array}{l}\text { Pentland, Brian T.; Feldman, Martha S.; } \\
\text { Becker, Markus C.; et al. }\end{array}$ & $\begin{array}{l}\text { Dinâmica de rotinas organizacionais. Modelo gerador de rotinas organizacionais. Os resultados sugerem que o foco na ação } \\
\text { fornece uma base útil e parcimoniosa para uma teoria de rotinas e capacidades organizacionais. }\end{array}$ \\
\hline & Turner, Scott F.; Fern, Michael J. & $\begin{array}{l}\text { Estabilidade e Variabilidade das Performances de rotina. Os efeitos da experiência e contexto Mudança. Micro fundações de } \\
\text { rotinas. }\end{array}$ \\
\hline & Teece, David J. & $\begin{array}{l}\text { Capacidade dinâmicas. Ação empreendedora. O papel dos executivos individuais no âmbito das capacidades dinâmicas. O } \\
\text { papel crítico do gerente empresarial tanto em transformar a empresa e moldar o ecossistema por meio de atos estratégicos. }\end{array}$ \\
\hline & $1 \mathrm{D}$. & $\begin{array}{l}\text { Ação de rotinas. Micro nível. Rotina é um modo distinto de ação organizacional. O papel dos artefatos na organização de } \\
\text { nossas ações. }\end{array}$ \\
\hline 2013 & $\begin{array}{l}\text { Loch, Christoph H.; Sengupta, Kishore; } \\
\text { Ahmad, M. Ghufran }\end{array}$ & $\begin{array}{l}\text { Microevolução de rotinas. Rotinas e resolução de problemas. Integração interna, identidade regulação grupo, distribuição de } \\
\text { status e relacionamentos. Dinâmicas de integração internas. }\end{array}$ \\
\hline
\end{tabular}


Rotinas Organizacionais no Ambiente da Teoria Evolucionária

\begin{tabular}{|c|c|c|}
\hline & Cohen, Lisa E. & $\begin{array}{l}\text { Um modelo de como as tarefas são agrupadas. Postos de trabalho. Projetos de trabalho e suas implicações de alcance. } \\
\text { Reconciliação, interpretação e performance. }\end{array}$ \\
\hline & $\begin{array}{l}\text { Tippmann, Esther; Mangematin, } \\
\text { Vincent; Scott, Pamela Sharkey }\end{array}$ & $\begin{array}{l}\text { Conhecimento organizacional. Busca de conhecimento para resolver problemas não rotineiros permite que gerentes de nível } \\
\text { médio, não só para conceber novas soluções, mas também para desenvolver capacidades organizacionais. }\end{array}$ \\
\hline \multirow{11}{*}{2014} & Canales, Rodrigo & $\begin{array}{l}\text { Gerenciando tensões organizacionais. Equilíbrio das pressões para alcance da eficiência. Capacidade estratégica. Micro } \\
\text { finanças. }\end{array}$ \\
\hline & Ioannou, Ioannis & $\begin{array}{l}\text { Performance das firmas advindas da empresa mãe. Aspectos positivos e negativos de desempenho organizacional advindo de } \\
\text { rompimento de rotinas. }\end{array}$ \\
\hline & $\begin{array}{l}\text { Vera, Dusya; Crossan, Mary; Rerup, } \\
\text { Claus; et al. }\end{array}$ & $\begin{array}{l}\text { Efeitos da "propensão ação individual". No sentido de resolver situações para as quais eles não têm o conhecimento e / ou } \\
\text { experiência sobre o que fazer. Conduta - 'pensar antes de agir "ou" agir antes de pensar " }\end{array}$ \\
\hline & Laureiro-Martinez, Daniella & $\begin{array}{l}\text { Bases cognitivas comportamentais. Rotinização. Tomada de Decisão. Três contribuições. Introdução e observação empírica } \\
\text { das capacidades de controle cognitivo. }\end{array}$ \\
\hline & D'Adderio, Luciana & Transferência de rotina. Efeitos da replicação na transferência de rotinas. \\
\hline & $\begin{array}{l}\text { Peeters, Carine; Massini, Silvia; Lewin, } \\
\text { Arie Y }\end{array}$ & O Papel das rotinas. Capacidade de absorção. \\
\hline & $\begin{array}{l}\text { Spedale, Simona; Coupland, Christine; } \\
\text { Tempest, Sue }\end{array}$ & $\begin{array}{l}\text { Contribuição para o estudo de preconceito de idade de gênero no local de trabalho por investigar como a rotina do dia-divisão } \\
\text { na radiodifusão participa na construção social de uma ideologia da juventude. }\end{array}$ \\
\hline & Bercovitz, Janet E. L.; Tyler, Beverly B. & $\begin{array}{l}\text { Como os papéis organizacionais direcionam a aprendizagem relacional de contratação de pessoal, o que influencia } \\
\text { posteriormente evolução contrato. Administradores de contratação e a proteção de conhecimento. }\end{array}$ \\
\hline & Whitford, Josh; Zirpoli, Francesco & Modelo pragmático de ação para a abordagem contemporânea "prática" para o estudo das organizações. \\
\hline & Brauer, Matthias; Laamanen, Tomi & $\begin{array}{l}\text { Força de trabalho e desempenho da empresa. Downsizing. Escassez de pesquisas sobre o que acontece quando as rotinas são } \\
\text { interrompidas ou dissolvida através da eliminação de indivíduos-chave envolvidos. }\end{array}$ \\
\hline & D'Adderio, Luciana; Pollock, Neil & O efeito das teorias organizacionais sobre a organização \\
\hline \multirow{7}{*}{2015} & Zheng, Yanfeng; Yang, Haibin & $\begin{array}{l}\text { Será que a familiaridade com parceiros de aliança promove inovações revolucionárias? Impacto e influência na repetição de } \\
\text { colaboração de rotinas interorganizacionais. }\end{array}$ \\
\hline & $\begin{array}{l}\text { Levinthal, Daniel A.; Marino, } \\
\text { Alessandro }\end{array}$ & $\begin{array}{l}\text { Mecanismos adaptativos, incluindo não só a possibilidade de aprendizagem e adaptação de um determinado comportamento, } \\
\text { mas também a seleção interna sobre alguma população de rotinas e comportamentos. Seleção, variedade e Plasticidade }\end{array}$ \\
\hline & $\begin{array}{l}\text { Eberl, Peter; Geiger, Daniel; } \\
\quad \text { Asslaender, Michael S. }\end{array}$ & $\begin{array}{l}\text { Reparação de confiança. Violação de integridade de nível organizacional. Regras organizacionais e confiabilidade. } \\
\text { Implementariedade das regras. }\end{array}$ \\
\hline & $\begin{array}{l}\text { Farjoun, Moshe; Ansell, Christopher; } \\
\text { Boin, Arjen }\end{array}$ & Filosofia e pragmatismo. \\
\hline & Barley, William C & $\begin{array}{l}\text { Os efeitos "representações durante e após a interação, caracterizando-os como ferramentas que ajudam a comunicar o } \\
\text { entendimento local com indivíduos que têm diferentes conhecimentos. }\end{array}$ \\
\hline & Patriotta, Gerardo; Gruber, Daniel A & Eventos planejados e inesperados e coordenação. \\
\hline & $\begin{array}{l}\text { Aaltonen, Aleksi; Lanzara, Giovan } \\
\text { Francesco }\end{array}$ & ernança. Capacidade e rotina. Aprendizagem individual e coletiva. \\
\hline
\end{tabular}




\begin{tabular}{|c|c|c|}
\hline & Busby, J. S.; Iszatt-White, M. & Regras regulativas e de segurança. A violação regra de rotina. \\
\hline & Heinze, Kathryn L.; Weber, Klaus & $\begin{array}{l}\text { Pluralismo organizacional. Intraempreendedorismo. Lógicas incumbentes estão entrincheirados em rotinas organizacionais. } \\
\text { Oportunismo do intraempreendedor. }\end{array}$ \\
\hline & $\begin{array}{l}\text { Helfat, Constance E.; Campo-Rembado, } \\
\text { Miguel A. }\end{array}$ & $\begin{array}{l}\text { Capacidade de integração. Comportamento organizacional em relação a integração vertical e capacidade de evolução. } \\
\text { Inovação sistêmica. }\end{array}$ \\
\hline & Wollersheim, Jutta; Heimeriks, Koen H. & Capacidades dinâmicas. \\
\hline & Siciliano, Michael & Controle organizacional. Experiências estéticas dos trabalhadores. \\
\hline & $\begin{array}{l}\text { Yi, Sangyoon; Knudsen, Thorbjorn; } \\
\text { Becker, Markus C. }\end{array}$ & Inercia de rotinas. Inércia nas rotinas e sua influência no processo de adaptação organizacional. \\
\hline & $\begin{array}{l}\text { Spee, Paul; Jarzabkowski, Paula; Smets, } \\
\text { Michael }\end{array}$ & Interdependência de rotinas. Coordenação de Rotinas. Padrões ostensivos. Rotinas de interseção. Reorientação de rotinas. \\
\hline & Sonenshein, Scott & Rotinas e criatividade. Rotinas como uma parte inerente da criatividade e criatividade como uma parte inerente de rotinas. \\
\hline & Sele, Kathrin; Grand, Simon & $\begin{array}{l}\text { Efeitos generativos mediadores pode levar a: (1) a criação de resultados inovadores, (2) a adaptação das performances } \\
\text { rotineiras existentes, e / ou (3) o surgimento de novas performances de rotina. Ecologia de rotinas. }\end{array}$ \\
\hline & $\begin{array}{l}\text { Kremser, Waldemar; Schreyoegg, } \\
\text { Georg }\end{array}$ & Inter-relações entre as rotinas organizacionais e seus efeitos sobre a dinâmica de rotina. Rotinas e clusters. \\
\hline 2016 & $\begin{array}{l}\text { Dittrich, Katharina; Guerard, Stephane; } \\
\text { Seidl, David }\end{array}$ & Papel da conversa reflexiva na forma de como as rotinas mudam. \\
\hline & $\begin{array}{l}\text { Deken, Fleur; Carlile, Paul R.; Berends, } \\
\text { Hans; et al. }\end{array}$ & Rotinas interdependentes e implicações. Análise de novo modelo de negócio de serviços com base na informação. \\
\hline & Danner-Schroeder, Anja; Geiger, Daniel & Padronização de trabalho e seus efeitos. Padrões de rotina reconhecidas como estáveis ou flexíveis. \\
\hline & Cohendet, Patrick S.; Simon, Laurent O. & $\begin{array}{l}\text { Eficiência criativa. Reconfiguração de rotinas. Aspecto ostensivo, aspecto formativo e artefatos de rotinas. Recombinar } \\
\text { aspectos de rotinas como maneira de alcance de equilíbrio. }\end{array}$ \\
\hline & Bucher, Silke; Langley, Ann & $\begin{array}{l}\text { Interrupção e reorientação de rotinas. O estudo oferece um modelo de mudança de rotina intencional que articula o papel dos } \\
\text { espaços em interromper e reorientar sua dinâmica recursiva. }\end{array}$ \\
\hline & $\begin{array}{l}\text { Bertels, Stephanie; Howard-Grenville, } \\
\text { Jennifer; Pek, Simon }\end{array}$ & Cultura e integração de rotinas. Moldagem cultural. Rotina como trégua. \\
\hline & $\begin{array}{l}\text { Berente, Nicholas; Lyytinen, Kalle; } \\
\text { Yoo, Youngjin; et al. }\end{array}$ & $\begin{array}{l}\text { Rotinas como amortecedores. Diferentes elementos de rotinas de ajustar dinamicamente ao longo do tempo para permitir a } \\
\text { implementação do sistema estável, aumentando a integração e controle de todo o organismo. }\end{array}$ \\
\hline & Aroles, Jeremy; McLean, Christine & $\begin{array}{l}\text { Estabilidade e mudança. Diferença e repetição. Rotinas padrão ou como problema na solução de ineficiência organizacional } \\
\text { Relações dinâmicas. }\end{array}$ \\
\hline & $\begin{array}{l}\text { LeBaron, Curtis; Christianson, Marlys } \\
\text { K.; Garrett, Lyndon; et al }\end{array}$ & Desempenho flexível no trabalho diário. Adaptação específica de rotina para cada tipo de situação. \\
\hline
\end{tabular}

Quadro 2 - apresentação de estudos encontrados na base Web Of Science (2009 - 2016) 


\section{CONSIDERAÇÕES FINAIS}

O presente trabalho buscou, a partir dos estudos do clássico de Nelson e Winter (2005) - "Uma Teoria Evolucionária da Mudança Econômica”, trazer reflexões sobre a trajetória dos estudos sobre rotinas organizacionais. Nesse sentido, vários aspectos relacionados à natureza do fenômeno e suas ambiguidades foram aqui apresentados. Entretanto, com certeza trabalhos importantes acerca do tema não foram aqui relacionados, em função das condições que cercam esse tipo de resenha.

Sobre esse debate, Becker (2002) defende que estudar a noção de Rotina Organizacional é tratar de complexidades, de elementos ambíguos, mas que são todos eles importantes para se conhecer economia e negócios e complementa “...não há um conceito individual que possa capturar todos eles. Se pudesse haver um conceito que pudesse capturá-los, ele teria que ser tão grande que, necessariamente, não teria nitidez". (Becker, 2002, p. 30)

Ao final desta breve reflexão sobre a noção de Rotinas Organizacionais com base na obra de Nelson e Winter (2005), não há dúvida que o debate sobre este tema apresenta um grande potencial de contribuição para estudos teórico-empíricos acerca das organizações. Não é por acaso que publicações acadêmicas de primeiro ranking no campo da Administração têm aberto espaços importantes para o tema rotinas, incluindo uma abordagem que procura captar o ambiente de rápidas mudanças denominada routines dynamics. (Special Issue on Routine Dynamics. Organization Science, 2016). O tema Rotinas Organizacionais ainda apresenta um imenso potencial para estudos e investigações no campo organizacional, especialmente no ambiente econômico atual, no qual a interação entre estabilidade e dinamismo constitui um paradoxo central

\section{REFERÊNCIAS}

Aaltonen, A, \& Lanzara, G. F. (2015). Building Governance Capability in Online Social Production: Insights from Wikipedia. Organization Studies, 36(12): 1649-1673.

Anand, G., Gray, J., \& Siemsen, E. (2012). Decay, Shock, and Renewal: Operational Routines and Process Entropy in the Pharmaceutical Industry. Organization Science, 23(6): 1700-1716.

Aroles, J, \& Mclean, C. (2016). Rethinking Stability and Change in the Study of Organizational Routines: Difference and Repetition in a Newspaper-Printing Factory. Organization Science, 27(3 Special Issue): 535-550.
Avery, J. F. (1996). The influence of positive and Negative Normative Feedback on the Development and, Persistence of Group Routines, $\mathrm{PhD}$ dissertation, Purdue University, Indiana, USA. Retrieved September 08, 2017 from: http://docs.lib.purdue.edu/dissertations/AAI972550 $5 /$.

Bapuji, H., Hora, M., \& Saeed, A. M. (2012). Intentions, Intermediaries, and Interaction: Examining the Emergence of Routines. Journal of Management Studies, 49(8 Special Issue): 15861607.

Barley, W. C. (2015). Anticipatory Work: How the Need to Represent Knowledge Across Boundaries Shapes Work Practices Within Them. Organization Science, 26(6): 1612-1628.

Becker, M., \& Knudsen, T. (2002). The role of routines in reducing uncertainty: some empirical evidence. Washington: Academy of Management Conference.

Becker, M. (2004). The concept of routines twenty years after Nelson and Winter (2005): a review of the literature. Industrial and Corporate Change, 4: 643-677.

Becker, M. C. (2008). Handbook of Organizational Routines. Cheltenham: Elgar.

Bercovitz, J. E. L., \& Tyler, B. (2014). Who I Am and How I Contract: The Effect of Contractors' Roles on the Evolution of Contract Structure in University-Industry Research Agreements. Organization Science, 25(6): 1840-1859.

Berente, N., Lyytinen, K, Yoo, Y., \& King, J.L. (2016). Routines as Shock Absorbers During Organizational Transformation: Integration, Control, and NASA's Enterprise Information System. Organization Science, 27(3 - Special Issue): 551-572.

Bertels, S, Howard-Grenville, J, \& Pek, S. (2016). Cultural Molding, Shielding, and Shoring at Oilco: The Role of Culture in the Integration of Routines. Organization Science 2 27(3 - Special Issue): 573593.

Brauer, M., \& Laamanen, T. (2014). Workforce Downsizing and Firm Performance: An Organizational Routine Perspective. Journal of Management Studies, 51(8): 1311-1333.

Brown, A. D., \& Lewis, M. A. (2011). Identities, Discipline and Routines. Organization Science, 32(7): 871-895. 
Bucher, S., \& Langley, A. (2016). The Interplay of Reflective and Experimental Spaces in Interrupting and Reorienting Routine Dynamics. Organization Science, 27(3 - Special Issue): 594-613.

Busby, J. S., \& Iszatt-White, M. (2016). Rationalizing Violation: Ordered Accounts of Intentionality in the Breaking of Safety Rules. Organization Studies, 37(1): 35-53.

Cabantous, L., \& Gond, J. P. (2011). Rational Decision Making as Performative Praxis: Explaining Rationality's Eternel Retour. Organization Science, 22(3): 573-586.

Cacciatori, E. (2012). Resolving Conflict in ProblemSolving: Systems of Artefacts in the Development of New Routines. Journal of Management Studies, 49(8 - Special Issue): 1559-1585.

Canales, R. (2014). Weaving Straw into Gold: Managing Organizational Tensions Between Standardization and Flexibility in Microfinance. Organization Science, 25(1): 1-28.

Christianson, M. K., \& Farkas, M. T., Sutcliffe, K. M., \& Weick, K. E. (2009). Learning Through Rare Events: Significant Interruptions at the Baltimore \& Ohio Railroad Museum. Organization Science, 20(5): 846-860.

Cohen, W. M., \& Bacdayan, P. (1994). Organizational routines are stored as procedural memory: evidence from a laboratory study. Organization Science, 5(4): $554-568$.

Cohen, W. M., Burkhart, R., Dosi, G., Egigi, M., Marengo, L., Warglien, M., \& Winter, S. (1996). Routines and other recurring action patterns of organizations: contemporary research issues. Industrial and Corporate Change, 5(3): 653-698.

Cohen, L. E. (2013). Assembling Jobs: A Model of How Tasks Are Bundled Into and Across Jobs. Organization Science, 24(2): 432-454.

Cohen, M. D. (2012). Perceiving and Remembering Routine Action: Fundamental Micro-Level Origins. Journal of Management Studies, 49(8 - Special Issue): 1383-1388.

Cohendet, P. S., \& Simon, L., O. (2016). Always Playable: Recombining Routines for Creative Efficiency at Ubisoft Montreal's Video Game Studio. Organization Science, 27(3 - Special Issue): 614-632.

Csaszar, F. A., \& Siggelkow, N. (2010). How Much to Copy? Determinants of Effective Imitation Breadth. Organization Science, 21(3): 661-676.
Currie, G., \& White, L. (2014). Inter-professional Barriers and Knowledge Brokering in an Organizational Context: The Case of Healthcare. Organization Studies, 33(10): 1333-1361.

D'adderio, L. (2014). The Replication Dilemma Unravelled: How Organizations Enact Multiple Goals in Routine Transfer. Organization Science, 25(5): 1325-1350.

D'adderio, L., \& Pollock, N. (2014). Performing Modularity: Competing Rules, Performative Struggles and the Effect of Organizational Theories on the Organization Organization Studies, 35(12): 1813-1843.

Danner-Schroeder, A., \& Geiger, D. (2016). Unravelling the Motor of Patterning Work: Toward an Understanding of the Microlevel Dynamics of Standardization and Flexibility. Organization Studies, 27(3 - Special Issue): 633-658.

Deken, F., Carlile, P. R., Berends, H., \& Lauche, K. (2016). Generating Novelty Through Interdependent Routines: A Process Model of Routine Work. Organization Science, 27(3 Special Issue): 659-677.

Dittrich, K., Guerard, S., \& Seidl, D. (2016). Talking About Routines: The Role of Reflective Talk in Routine Change. Organization Science, 27(3 Special Issue): 678-697.

Eberl, P., Geiger, D., \& Asslaender, M. S. (2015). Repairing Trust in an Organization after Integrity Violations: The Ambivalence of Organizational Rule Adjustments. Organization Studies, 36(9 Special Issue): 1205-1235.

Eisenhardt, K. M., Furr, N. R., \& Bingham, C. B. (2010). Microfoundations of Performance: Balancing Efficiency and Flexibility in Dynamic Environments. Organization Science, 21(6): 12631273.

Farjoun, M., Ansell, C., \& Boin, A. (2015). Pragmatism in Organization Studies: Meeting the Challenges of a Dynamic and Complex World. Organization Science, 26(6): 1787-1804.

Feldman, M.S. (2000). Organizational Routines as a Source of Continuous Change, Organization Science, 11(6): 611-629.

Feldman, M. S., \& Pentland, B. T. (2003). A performative perspective on stability and change in organizational routines. Industrial and Corporate Change, 12: 727-752. 
Feldman, M. S., \& Pentland, B. T. (2003). Reconceptualizing Organizational Routines as a Source of Flexibility and Change, Administrative Science Quarterly, 48: 94-118.

Feldman, M. S., \& Orlikowski, W. J. (2011). Theorizing Practice and Practicing Theory. Organization Science, 22(5): 1240-1253.

Feldman, M. S., Pentland, B. T., D'Adderio, L., \& Lazaric, N. (2016). Beyond Routines as Things: Introduction to the Special Issue on Routine Dynamics. Organization Science, 27(3): 505-513.

Heinze, K. L., \& Weber, K. (2016). Toward Organizational Pluralism: Institutional Intrapreneurship in Integrative Medicine. Organization Science, 27(1): 157-172.

Helfat, C. E., \& Campo-Rembado, M. A. (2016). Integrative Capabilities, Vertical Integration, and Innovation Over Successive Technology Lifecycles. Organization Science, 27(2): 249-264.

Hodgson, G. M. (1997). The ubiquity of habits and rules. Cambridge Journal of Economics, 21(6): 663-684

Ioannou, I. (2014). When Do Spinouts Enhance Parent Firm Performance? Evidence from the US Automobile Industry, 1890-1986. Organization Science, 25(2): 529-551.

Jarzabkowski, P. A., Le, J. K., \& Feldman, M. S. (2012). Toward a Theory of Coordinating: Creating Coordinating Mechanisms in Practice. Organization Science, 23(4): 907-927.

Jones, O., \& Craven, M. (2001). Expanding capabilities in a mature manufacturing firm: Absorptive capacity and the TCS. International Small Business Journal, 19(3): 39-55.

Kane, A A. (2010). Unlocking Knowledge Transfer Potential: Knowledge Demonstrability and Superordinate Social Identity. Organization Science, 21(3): 643-660.

Knott, A. M., \& Mckelvey, B. (1999). Nirvana, Efficient: a comparative test of residual claims and routines. Journal Economics Behavioral on Organization, 88: $365-383$.

Kremser, W., \& Schreyoegg, G. (2016). The Dynamics of Interrelated Routines: Introducing the Cluster Level. Organization Science,_27(3 - Special Issue): 698-721.
Laamanen, T., \& Wallin, J. (2009). Cognitive Dynamics of Capability Development Paths. journal Of Management Studies, 46(6): 950-981.

Labatut, J., Aggeri, F., \& Girard, N. (2012). Discipline and Change: How Technologies and Organizational Routines Interact in New Practice Creation. Organization Studies, 33(1): 39-69.

Laureiro-Martinez, D. (2014). Cognitive Control Capabilities, Routinization Propensity and Decision-Making Performance. Organization Science, 25(4): 1111-1133.

Lavie, D., Kang, J., \& Rosenkopf, L. (2011). Balance Within and Across Domains: The Performance Implications of Exploration and Exploitation in Alliances. Organization Science, 22(6): 1517-1538.

Lebaron, C., Christianson, M. K., Garrett, L., \& Ilan, R. (2016). Coordinating Flexible Performance During Everyday Work: An Ethnomethodological Study of Handoff Routines. Organization Science, 27(3 - Special Issue): 514-534.

Leonardi, P. M. (2011). Innovation Blindness: Culture, Frames, and Cross-Boundary Problem Construction in the Development of New Technology Concepts. Organization Science, 22(2): 347-369.

Levinthal, D. A., \& Marino, A. (2015). Three Facets of Organizational Adaptation: Selection, Variety, and Plasticity. Organization Science, 26(3): 743-755.

Lewin, A. Y., Massini, S., \& Peeters, C. (2011). Microfoundations of Internal and External Absorptive Capacity Routines. Organization Science, 22(1): 81-98.

Levitt, B., \& March, J. G. (1988). Organizational learning. Annual Review of Sociology, 14: 319-340.

Loch, C. H., Sengupta, K., \& Ahmad, M. G. (2013). The Microevolution of Routines: How Problem Solving and Social Preferences Interact. Organization Science, 24(1): 99-115.

March, J. G., \& Simon, H. A. (1958). Organizations. New York: John Wiley \& Sons.

March, J. G. \& Olsen, J. P. (1976). Ambiguity and Choice in Organization. Bergen: Universitetsforlaget.

Mathews, J. A. (2010). Lachmannian Insights into Strategic Entrepreneurship: Resources, Activities and Routines in a Disequilibrium World. Organization Studies, 31(2): 219-244. 
Mckendrick, D. G., Wade, J. B., \& Jaffee, J. (2009). A Good Riddance? Spin-Offs and the Technological Performance of Parent Firms. Organization Science, 20(6): 979-992.

Miller, K. D., Pentland, B. T., \& Choi, S. (2012). Dynamics of Performing and Remembering Organizational Routines. Journal of Management Studies, 49(8- Special Issue): 1536-1558.

Nelson, R. R. (1994), Routines In G. Hodgson, W. Samuls, \& M. Tool (Ed.). The Elgar Companion to Institutional and Evolutionary Economics (Vol. 2, pp. 249-253). Edward Elgar: Aldershot.

Nelson, R. R., \& Winter, S. G. (2005). Uma Teoria Evolucionária da Mudança Econômica. Campinas: Editora da Unicamp.

Nicolini, D. (2011). Practice as the Site of Knowing: Insights from the Field of Telemedicine. Organization Science,_22(3): 602-620.

Obstfeld, D. (2012). Creative Projects: A Less Routine Approach Toward Getting New Things Done. Organization Science, 23(6):, 1571-1592.

Patriotta, G., \& Gruber, D. A. (2015). Newsmaking and Sensemaking: Navigating Temporal Transitions Between Planned and Unexpected Events. Organization Science, 26(6): 1574-1592.

Peeters, C., Massini, S. \& Lewin, A Y. (2014). Sources of Variation in the Efficiency of Adopting Management Innovation: The Role of Absorptive Capacity Routines, Managerial Attention and Organizational Legitimacy. Organization Studies, 35(9 - Special Issue): 1343-1371.

Pentland, B., \& Rueter, H. (1994). Organizational Routines as Grammars of Action. Administrative Science Quarterly, 39: 484-510.

Pentland, B. T., Haerem, T., \& Hillison, D. (2010). Comparing Organizational Routines as Recurrent Patterns of Action. Organization Studies_31(7): 917-940.

Pentland, B. T., Haerem, T., \& Hillison, D. (2011). The (N)Ever-Changing World: Stability and Change in Organizational Routines. Organization Science, 22(6): 1369-1383.

Pentland, B. T., Feldman, M. S., Becker, M. C., \& Liu, P. (2012). Dynamics of Organizational Routines: A Generative Model. Journal of Management Studies, 49(8 - Special Issue): 1484-1508.

Postrel, S., \& Rumelt, R. P. (1992). Incentives, Routines, and Self-Command. Industrial and Corporate Change, 1: 397-425.
Raman, R., \& Bharadwaj, A. (2012). Power Differentials and Performative Deviation Paths in Practice Transfer: The Case of Evidence-Based Medicine. Organization Science, 23(6): 1593-1621.

Reynaud, B. (1998). Les propriétés des Routines: Outils Pragmatiques de Décision et Models de Coordination Collective, Sociologie du travail, 4: 465477

Rennstam, J. (2012). Object-Control: A Study of Technologically Dense Knowledge Work. Organization Studies, 33(8): 1071-1090.

Salvato, C. (2009). Capabilities Unveiled: The Role of Ordinary Activities in the Evolution of Product Development Processes. Organization Science, 20(2): 384-409.

Schreyoegg, G., \& Sydow, J. (2011). Organizational Path Dependence: A Process View. Organization Studies,_32(3), 321-335.

Sele, K., \& Grand, S. (2016). Unpacking the Dynamics of Ecologies of Routines: Mediators and Their Generative Effects in Routine Interactions. Organization Science, 27(3 - Special Issue): 722738 .

Siciliano, M. (2016). Disappearing into the Object: Aesthetic Subjectivities and Organizational Control in Routine Cultural Work. Organization Studies, 37(5): 687-708.

Simon, H. A. (1997). Administrative Behavior: A Study of Decision-Making Processes in Administrative Organizations (4th ed.). New York: The Free Press.

Simon, H. A. (1955). A Behavioral Model of Rational Choice. Quaterly Journal of Economics, 69: p.99118.

Sminia, H. (2011). Institutional Continuity and the Dutch Construction Industry Fiddle. Organization Studies, 32(11): 1559-1585.

Sonenshein, S. (2016). Routines and Creativity: From Dualism to Duality. Organization Science, 27(3 Special Issue): 739-758.

Spedale, S., \& Coupland, C. (2014). Tempest, S. Gendered Ageism and Organizational Routines at Work: The Case of Day-Parting in Television Broadcasting. Organization Studies, 35(11 Special Issue): 1585-1604.

Spee, P., Jarzabkowski, P., \& Smets, M. (2016). The Influence of Routine Interdependence and Skillful Accomplishment on the Coordination of 
Standardizing and Customizing. Organization Science, 27(3 - Special Issue): 759-781.

Sullivan, B. (2010). Competition and Beyond: Problems and Attention Allocation in the Organizational Rulemaking Process. Organization Science, 21(2): 432-450.

Teece, D. J., Rumelt, R., Dosi, G., \& Winter, S. G. (1994). Understanding corporate coherence: Theory and evidence. Journal of Economic Behavior and Organization, 23: 1-30.

Teece, D. J. (2012). Dynamic Capabilities: Routines versus Entrepreneurial Action. Journal of Management Studies, 49(8- Special Issue): 13951401.

Tippmann, E., Mangematin, V., \& Scott, P. S. (2013). The Two Faces of Knowledge Search: New Solutions and Capability Development. Organization Studies, 34(12): 1869-1901.

Turner, S. F., \& Fern, M. J. (2012). Examining the Stability and Variability of Routine Performances: The Effects of Experience and Context Change. Journal of Management Studies, 49(8 - Special Issue): 1407-1434.

Turner, S. F., \& Rindova, V. (2012). A Balancing Act: How Organizations Pursue Consistency in Routine Functioning in the Face of Ongoing Change. Organization Science, 23 (1): 24-46.

Vera, D., Crossan, M., Rerup, C., \& Werner, S. (2014). 'Thinking Before Acting' or 'Acting Before Thinking': Antecedents of Individual Action Propensity in Work Situations. Journal of Management Studies, 51(4): 603-633.

Weick, K. E. (1990). The Vulnerable System: an analysis of the Tenerife air disaster. Journal of Management, 16: 571 - 593.

Whitford, J; Zirpoli, F. (2014). Pragmatism, Practice, and the Boundaries of Organization. Organization Science, 25(6): 1823-1839.

Winter, S. G. (1986) The Research Program of the Behavioral Theory of the Firm: Orthodox Critique and Evolutionary Perspective In B. Gilad \& S.
Kaish (Ed). Handbook of Behavioral Economics (Vol. A, pp. 151-188). Greenwich: JAI Press.

Winter, S. G. (1987a) Knowledge and Competence as Strategic Assets In D. J. Teece (Ed.). The Competitive Challenge - Strategies for Industrial Innovation and Renewa (pp. 159-184). Cambridge: Ballinger

Winter, S. G. (1987b). Natural selection and evolution In J. Eatwell, M. Milgate, \& P. Newman (Ed.). The New Palgrave of Economics (Vol. 3, pp. 614-617). London: Macmillan.

Winter, S. G. (1994). Organizing for Continuous Improvement: Evolutionary Theory Meets the Quality Revolution. In J. A. C. Baum \& J. V. Singh (Ed.). Evolutionary Dynamics of Organization (Chap. 6, pp. 90-118). Oxford: Oxford University Press.

Wollersheim, J., \& Heimeriks, K. H. (2016). Dynamic Capabilities and Their Characteristic Qualities: Insights from a Lab Experiment. Organization Science, 27(2): 233-248.

Yi, S., Knudsen, T., \& Becker, M. C. (2016). Inertia in Routines: A Hidden Source of Organizational Variation. Organization Science, 27(3 - Special Issue): 782-800.

Zahara, S. A., \& George, G. (2002). Absorptive capacity: A review, reconceptualization, and extension. The Academy of Management Review, 27(2): 185-203.

Zbaracki, M. J., \& Bergen, M. (2010). When Truces Collapse: A Longitudinal Study of PriceAdjustment Routines. Organization Science, 21(5): 955-972.

Zheng, Y., \& Yang, H. (2015). Does Familiarity Foster Innovation? The Impact of Alliance Partner Repeatedness on Breakthrough Innovations. Journal of Management Studies, 52(2): 213-230.

Zollo, M., \& Winter, S. (2002). Deliberate learning and the evolution of dynamics capabilities. Organization Science, 13(3): 339-351. 